\title{
Large area magneto-optical investigations of YBCO thin films
}

\author{
M. Kuhn, ${ }^{a}$ B. Schey, W. Biegel, and B. Stritzker \\ Universität Augsburg, Institut für Physik, D-86135 Augsburg, Germany \\ J. Eisenmenger and P. Leiderer \\ Universität Konstanz, Fakultät für Physik, D-78434 Konstanz, Germany
}

(Received 22 September 1998; accepted for publication 25 November 1998)

\begin{abstract}
A new apparatus for magneto-optical investigations of high temperature superconducting (HTS) films as large as $20 \times 20 \mathrm{~cm}^{2}$ is presented. With this equipment flux penetration of an external magnetic field into YBCO thin films has been studied by scanning the samples through an inhomogeneous magnetic field (magneto-optical scanning technique, MOST). The normal penetration of magnetic flux into a superconductor will be changed drastically in the presence of defects. The apparatus was constructed to realize an effective quality control of large area HTS thin films used for device fabrication. With this technique, a visualization of flux pattern in superconducting films larger than $1 \times 1 \mathrm{~cm}^{2}$ is presented for the first time. The results are compared to inductive $j_{c}$ measurements as well as to micrographs [optical microscope (OM), scanning electron microscopy (SEM)] and show that also in the large area characterization the magneto-optical method is very sensitive to microstructural defects impairing the critical current density, which is the relevant parameter for an application of the superconducting thin films. Moreover, it could be shown that MOST has appreciable advantages compared to inductive $j_{c}$ scans and microscopy (OM, SEM). In particular it is possible to observe defects, which are below the optical resolution of the MOST setup. (C) 1999 American Institute of Physics.
\end{abstract}

[S0034-6748(99)02103-6]

\section{INTRODUCTION}

The most common methods for nondestructive characterizations of a superconductor with regard to its magnetic and electric properties are alternating current susceptibility measurements, inductive methods, or vibrating sample magnetometry. All these methods are only sensitive to the total magnetic moment of the sample integrated over a larger area on the $\mathrm{mm}^{2}$ scale.

An alternative method which allows a direct visualization of the dynamic magnetic flux penetration into a superconductor is the magneto-optical microscopy ${ }^{1-5}$ based on the Faraday effect. This technique was first used for investigations of low temperature superconductors. ${ }^{1-4}$ Since the discovery of the high temperature superconductor (HTS), the technique was improved for investigations of HTS thin films and single crystals. The magneto-optical indicators used mostly in recent years were $E u \mathrm{~S}, E u \mathrm{~F}_{2}$, and $E u \mathrm{Se}^{6-9}$ for the low temperature range $(<20 \mathrm{~K})$, which could be also evaporated directly onto the superconductor resulting in a high lateral resolution $[\approx 0.5 \mu \mathrm{m}$, high resolution Faraday (HRF) technique]. ${ }^{6,79}$ With these indicators, also the flux dynamic in the nanosecond-range was investigated. ${ }^{10,11}$ For the higher temperature range (up to $77 \mathrm{~K}$ ) iron-garnet (IG) films were used as indicators showing the Faraday effect up to room temperature. Moreover they have a large Faraday rotation resulting in a high contrast, as well as a high magnetic sensitivity $\left(\geqslant 10 \mu \mathrm{T}^{12}\right)$. However these films have to be grown

${ }^{\text {a)} E l e c t r o n i c ~ m a i l: ~ m a r c u s . k u h n @ p h y s i k . u n i-a u g s b u r g . d e ~}$ on special substrates, they could not be evaporated directly onto the superconductor. The first garnet films showed a stripe-like pattern caused by their magnetic domains oriented perpendicular to the film surface. ${ }^{12,13}$ This pattern was superimposing the observation of the flux pattern caused by the superconductor and reducing the resolution to the scale of the domain width $(\approx 2-5 \mu \mathrm{m}$ at $0 \mathrm{~T})$. Recently IG films with in-plane anisotropy ${ }^{14-17}$ have been used. The spatial resolution here is determined by the film thickness and the distance between the garnet film and the superconductor, because the garnets grown on a separate substrate are just put on top of the superconductor. With these kinds of IG films, a high temperature observation of the flux distribution in a superconductor without disturbing domain pattern is possible.

The magneto-optical technique allows an investigation of the homogeneity of superconductors and of the influence of defects on the current distribution because it is very sensitive to defect detection. ${ }^{18,19}$

One limiting factor depending on the size of the investigated areas so far was the application of a homogeneous magnetic field. Thus, the investigated superconducting thin films typically have dimensions $\leqslant 1 \times 1 \mathrm{~cm}^{2}$. First experiments with an inhomogeneous magnetic field applied to small samples were carried out by J. Eisenmenger et al. ${ }^{16}$ and Govorkov et al. ${ }^{20}$ An experimental setup with an inhomogeneous magnetic field allows the investigation of large area HTS thin films via magneto-optical scanning technique (MOST). In the following chapter an apparatus for MOST is presented. 


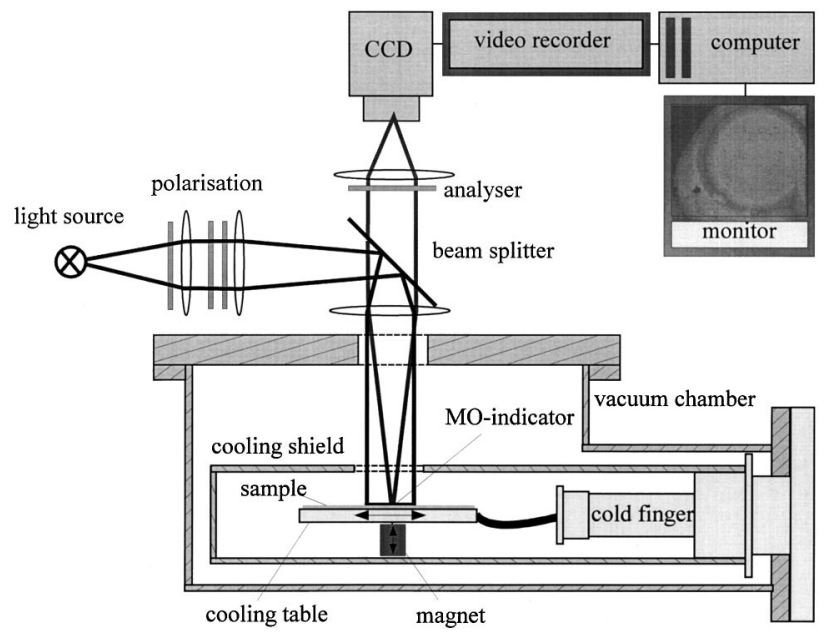

FIG. 1. Experimental setup of the MOST for investigations of large area superconducting thin films. The cooling table can pick up samples as large as $20 \times 20 \mathrm{~cm}^{2}$ and is scanned in two directions. The optical setup, the magneto-optical indicator, and the magnet, are kept in a fixed position.

\section{EXPERIMENT}

The basic idea of MOST is the movement of the sample within a fixed magneto-optical device consisting of a magnetic field, a magneto-optically active film, and the illuminating and imaging unit.

\section{A. Faraday effect}

The technique used for the visualization of the flux pattern in superconductors is based on the Faraday effect of a magneto-optically active layer. This layer is illuminated by linearly polarized light. In the presence of a magnetic field $H$ parallel to the propagation direction, the polarization vector of the incident light will be rotated by an angle $\varphi \propto H$ while passing through the magneto-optical film.

For an improved light reflection, a mirror layer is evaporated onto the magneto-optical indicator directed towards the superconducting thin film. After reflection and passing the indicator again, only light with a twisted polarization vector can pass the analyzer, which is in crossed position to the polarizer (Figs. 1 and 2).

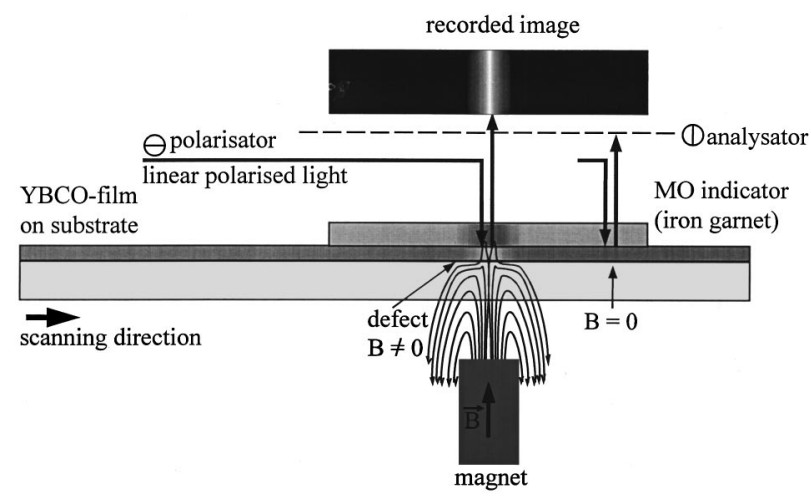

FIG. 2. Principle of magneto-optical image recording. The magnetooptically active layer (iron garnet) is illuminated by linearly polarized light. A magnetic field rotates the polarization vector by an angle $\varphi \propto H$.

\section{B. Apparative setup}

To pick up samples with dimensions as large as $20 \times 20$ $\mathrm{cm}^{2}$, the vacuum chamber (Fig. 1) includes a $\mathrm{Cu}$ table of
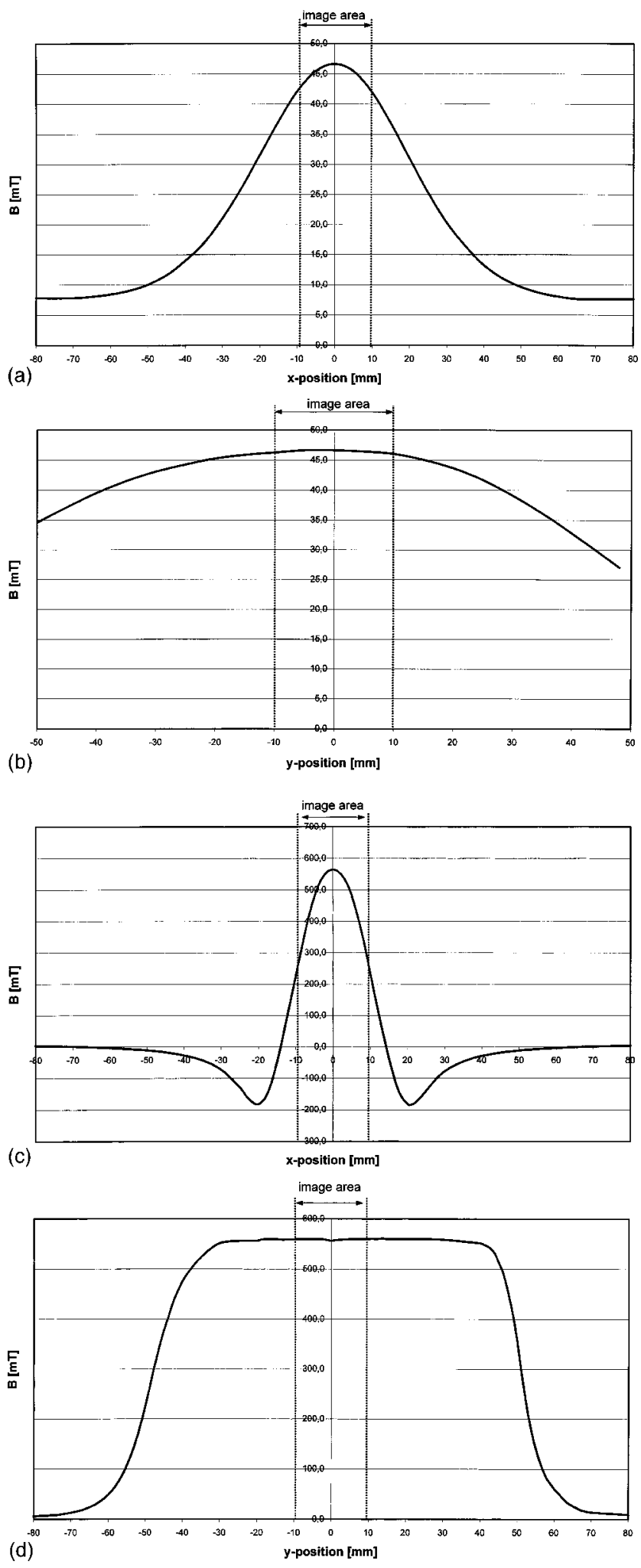

FIG. 3. Magnetic field strength of the magnet without superconducting sample in the (a), (c) $x$ - and (b), (d) $y$ direction measured by a Hall probe at a position on the film surface. The distance magnet-film surface was in (a), (b) $43.5 \mathrm{~mm}$ and corresponds to the distance Fig. 5(a) was recorded. In [Figs. 3(c) and 3(d)] the distance was $5.5 \mathrm{~mm}$. The dotted lines indicate the observation range for image recording. 
$26 \times 32 \mathrm{~cm}^{2}$ thermally coupled to a cold finger. The magnetooptical measurements can be carried out in a temperature range from $45-90 \mathrm{~K}$. The cooling is performed by a Gifford-McMahon-refrigerator system.

The whole sample area can be investigated by scanning the table in two directions. Since the sample is moved, the three other necessary parts for flux visualization, the optical setup, the magneto-optical indicator, and the magnet are kept at a fixed position under the optical window of the chamber (Fig. 2). The optical setup is built based on the illumination principle of Koehler. A foil is used as a polarizer, as well as an analyzer. The best contrast was achieved with the polarizer parallel to the light incident plane at the beam splitter and the analyzer turned $10^{\circ}-15^{\circ}$ out of the crossing position. The flux penetration into the superconductor can be visualized by the magneto-optical indicator illuminated with the polarized light perpendicular to the film surface. The flux pattern results in a gray scale picture recorded by a charge coupled device $(\mathrm{CCD})$ camera $($ EHDkam02E, $752 \times 582$ pixels) on a video tape (S-VHS Metz 64VA14). Single pictures can be grabbed by a computer so that a sequence of magneto-optical images is obtained showing the scanning process with an applied magnetic field. They can also be composed to a map of the samples indicating possibly present defects.

\section{Magneto-optical indicator}

Bismuth- and gallium-doped lutetium-iron-garnet films $\left[(\mathrm{Lu}, \mathrm{Bi}, \mathrm{Ga})_{3} \mathrm{Fe}_{5} \mathrm{O}_{12}\right]$ with an in-plane anisotropy were used as a magneto-optical indicator. The garnet films were grown on a (111)-gadolinium-gallium-garnet-substrate by liquid phase epitaxy. ${ }^{21-23}$
Because of the limited size of the available indicators $\left(\leqslant 3^{\prime \prime}\right)$ in relation to the superconductor, the sample has to be moved under the indicator. As a result, a larger gap between the garnet and the sample exists causing a lower optical resolution. In our case, the spatial resolution is about $50 \mu \mathrm{m}$. But since this technique is a magneto-optical one not only the optical resolution, but also the magnetic sensitivity is important. With the used IG indicators, a very high magnetic sensitivity in the $\mu \mathrm{T}$ range is obtained. Hence, defects below the optical resolution of our setup can be detected. The following experiments show that the technique is indeed a very useful method for studying the homogeneity of a superconductor in a complete manner combining flux visualization of a large sample area with an intermediate optical resolution and a very high magnetic sensitivity.

\section{Magnetic field}

To realize a magneto-optical investigation of large area superconducting thin films, an inhomogeneous magnetic field is used. This inhomogeneous field is created by a rectangular block of a permanent magnet (SmCo), whose magnetic moments are oriented perpendicular to the top side. The field can be increased up to $560 \mathrm{mT}$ at the surface of the sample by changing the distance between magnet and sample. The present magnet geometry was used to realize an investigation of a whole $20 \times 20 \mathrm{~cm}^{2}$ sample without gaps in three $20 \times 7 \mathrm{~cm}^{2}$ scans. Therefore, the magnet has a strong field gradient in one direction [Figs. 3(a) and 3(b)] and is nearly homogeneous over a larger range of $7 \mathrm{~cm}$ in the perpendicular direction [Figs. 3(c) and 3(d)]. Depending on the type of magnet and the distance to the sample, the field can be varied up to $560 \mathrm{mT}$ in maximum. For stronger fields, the
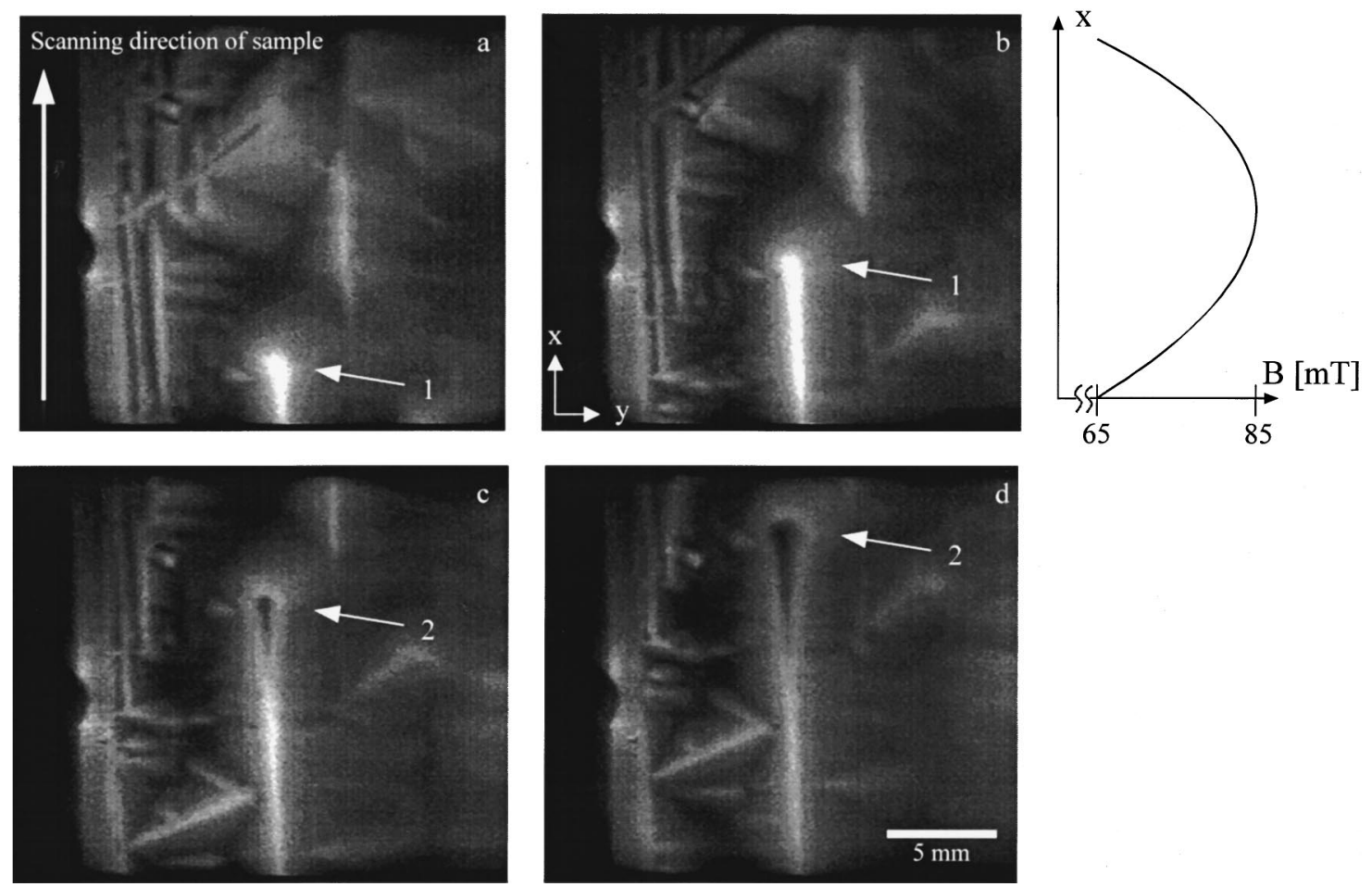

FIG. 4. Magneto-optical images recorded at $50 \mathrm{~K}$ indicating the dynamic of the MOST at one linear defect parallel to the scanning direction. The magnetic field distribution is shown in the graph on the right. The scanning direction is in the $x$ direction. 
gradient of the field increases as well [Figs. 3(a) and 3(c)]. In the case of a completely homogeneous magnetic field, the penetration of vortices depends strongly on the sample geometry. ${ }^{24,25}$ This can be suppressed by using an inhomogeneous field. ${ }^{16,20}$ Our intermediate case has to be considered by investigations of samples with sizes in the dimension of the magnet extension. Hence, an influence of sample geometry on the flux pattern should be taken into account.

\section{E. HTS samples}

The epitaxial YBCO thin films used for investigations were prepared by pulsed laser deposition ${ }^{26}$ on different substrates $(\mathrm{MgO}, \mathrm{YSZ}){ }^{27}$ The film thickness is typically about $150 \mathrm{~nm}$ and the lateral dimensions were 2 in. and $7 \times 7 \mathrm{~cm}^{2}$ in this case. The critical temperature $T_{c}$ of the different films was 88.0 and $90.3 \mathrm{~K}$, respectively, and the critical current density $j_{c}$ varied from $0.3-2.5 \times 10^{6} \mathrm{~A} / \mathrm{cm}^{2}$ at $77 \mathrm{~K}$ and zero field. To study the potential of the new apparatus in large area investigations of HTS thin films, samples with intrinsic and artificial defects have been used in the presented experiments.

\section{RESULTS AND DISCUSSION}

According to the literature up to now, magneto-optical studies of flux patterns were limited to superconducting thin films with a standard dimension of about $1 \times 1 \mathrm{~cm}^{2}$. With our new apparatus, it is possible to image flux distributions in HTS samples as large as $20 \times 20 \mathrm{~cm}^{2}$. Using the above mentioned special magnetic geometry, which has a strong field gradient along one direction and a nearly homogeneous range along the other direction, flux penetration depends strongly on the dimensions of the samples. Penetration into small films $\left(1 \times 1 \mathrm{~cm}^{2}\right)$ is similar to that observed with a homogeneous magnetic field starting simultaneously from all film edges. In the case of large area samples, field penetration starts in the area of maximum magnetic field. Thus, it depends on the magnet position before starting the scanning. If the scanning starts in the center of the sample, the penetration starts preferentially at defects and areas of lower critical current density, i.e., at areas with a lower $\mathrm{B}_{\mathrm{c} 1}$.

The sequence of pictures in Figs. 4(a)-4(d) shows a part of the magneto-optical investigations of a $7 \times 7 \mathrm{~cm}^{2}$ YBCO sample at $50 \mathrm{~K}$ and a magnetic field of $85 \mathrm{mT}$ in maximum. Bright areas indicate penetrated flux and dark contrast shows domains remaining in the Meissner phase. A typical flux penetration process is imaged at a defect (marked by the arrows) with an extension parallel to the scanning direction of the sample, that means also parallel to the strong gradient ( $x$ direction) of the inhomogeneous magnetic field which is about $\pm 20 \mathrm{mT}$ in this case. The field distribution in the $x$ direction is indicated on the right side, as well as the scanning direction of the sample [Fig. 4(a)]. In front of the maximum field, vortices enter the line-like defect [Figs. 4(a) and 4(b): bright areas, arrow 1] with increasing field and from there spread out into the sample. A brighter contrast of the image indicates that a larger magnetic field can penetrate the defect at this position. Behind the field maximum, where the field is decreasing again, flux starts to leave the defect indi-

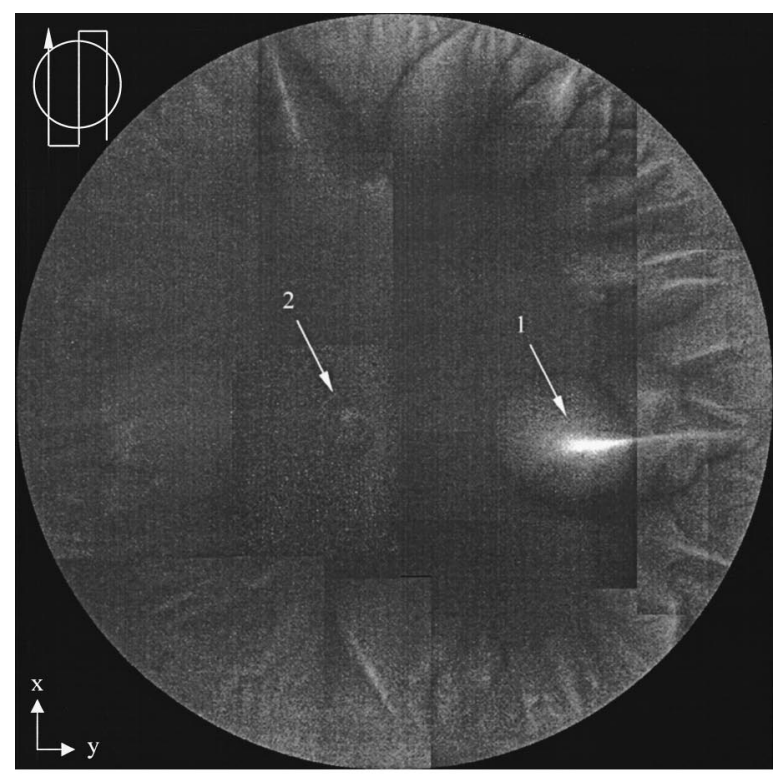

(a)

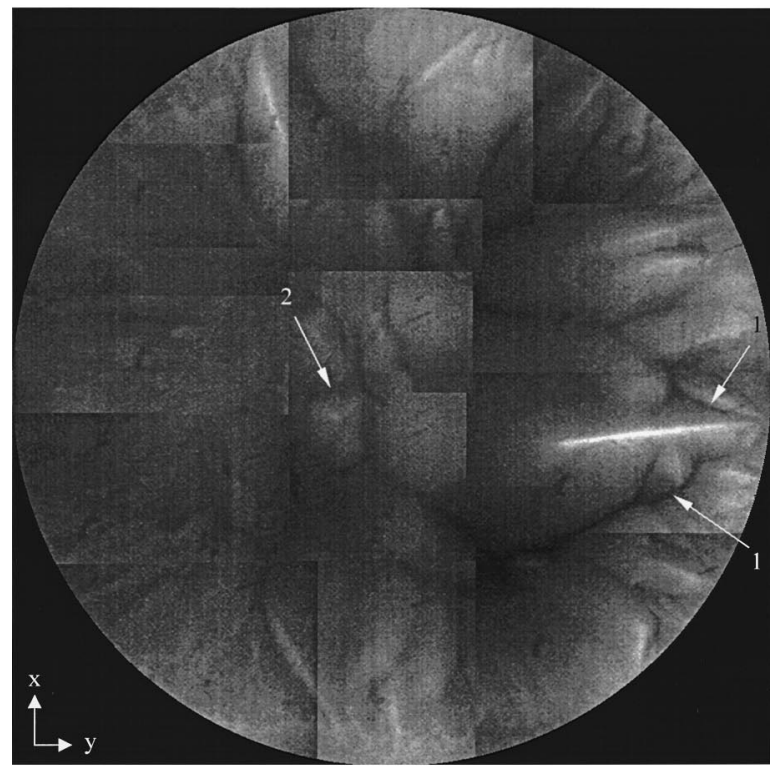

(b)

FIG. 5. Flux distribution in a 2 in. YBCO thin film visualized magnetooptically at $50 \mathrm{~K}$. The applied maximum magnetic field is (a) $45 \mathrm{mT}$ (corresponding to the field distribution in Fig. 3) and (b) $105 \mathrm{mT}$. The $d^{+}$lines are indicated in (b) by the remaining dark lines (arrows). Inset in (a): scanning process during the measurement of a 2 in. sample.

cated as dark areas between the white frame of the flux front [Figs. 4(c) and 4(d): arrow 2]. Therefore it is possible to observe the process of flux entering and exiting the sample in one picture showing the superconductor in different states of magnetization.

The results of a 2 in. YBCO sample investigated at $50 \mathrm{~K}$ is shown in Figs. 5(a) and 5(b). The two pictures show magneto-optical images of the flux distribution in the $2 \mathrm{in}$. YBCO thin film in a magnetic field of 45 and $105 \mathrm{mT}$ in maximum, respectively, after field cooling in the stray field $(<10 \mathrm{mT})$ of the magnet in this case. Both pictures represent a "qualitative map of defects" present in the superconducting thin film. A whole picture is composed of several single 


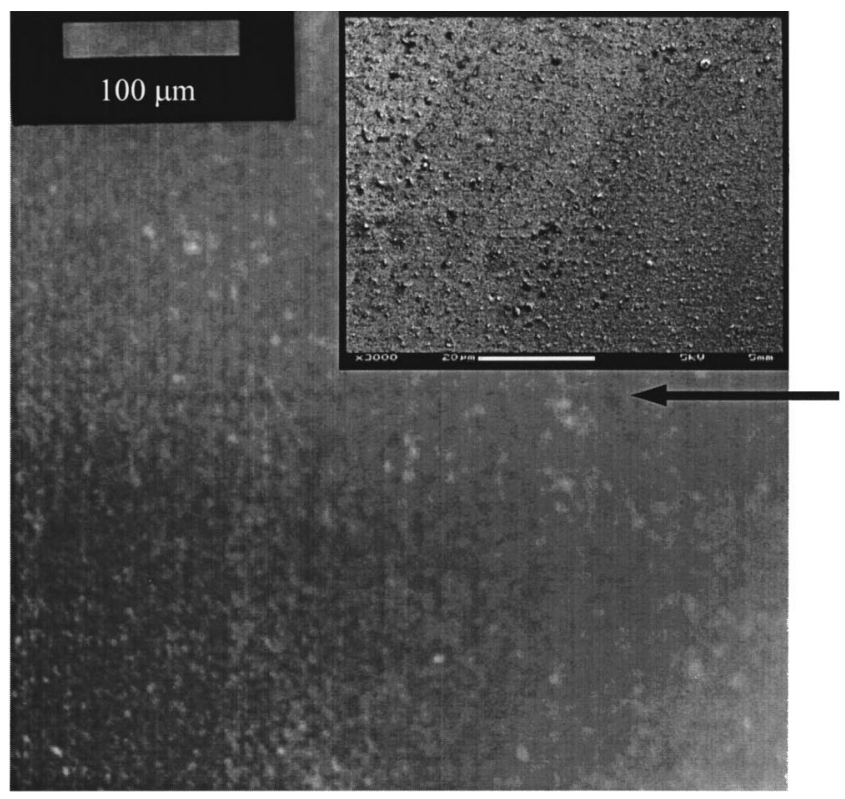

(a)

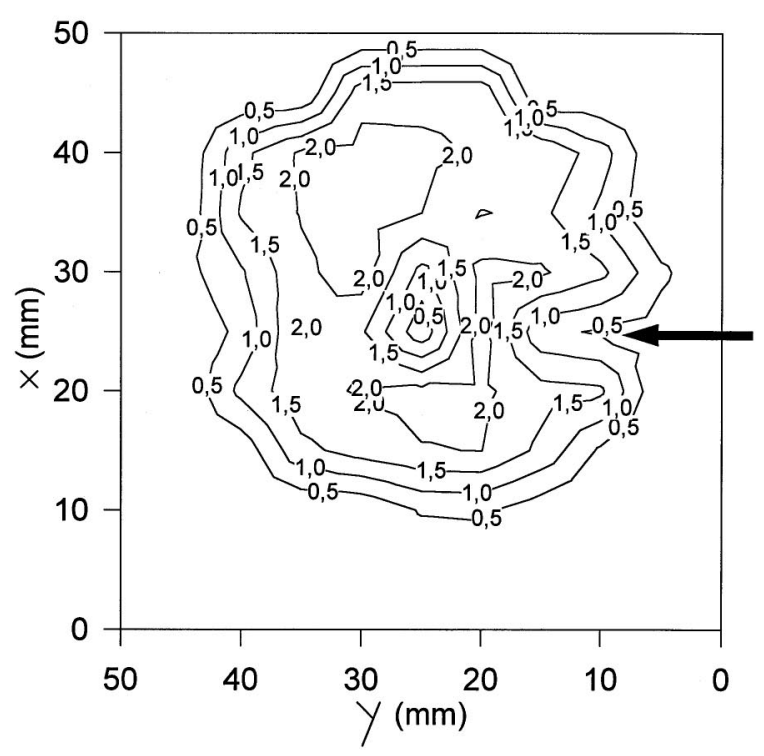

(b)

FIG. 6. (a) Optical micrograph of the scratch (arrow) detected magneto-optical on the 2 in. sample in Fig. 5. The scratch has a width of $5 \mu$ m. Inset in (a): SEM image of the scratch area. No defect could be observed (the white line indicates $20 \mu \mathrm{m}$ ). (b) Inductive $j_{c}$ scan of the $2 \mathrm{in}$. YBCO thin film in a $0.5 \times 0.5$ $\mathrm{cm}^{2}$ screen. The critical current density varies from 0.5 to $2.5 \mathrm{MA} / \mathrm{cm}^{2}$. The extended linear defect can be seen at the right-hand side (arrow).

images recorded dynamically during a $x$ - and $y$ scan over the whole sample area in three tracks through the strong magnetic field gradient in the $x$ direction [inset Fig. 5(a)]. The magnetic field specified for each picture is the maximum of the applied external field. Therefore, the flux pattern resulting from defects present in the sample becomes more complicated because of the inhomogeneous magnetic field. Nevertheless a qualitative statement of the defect structure in the superconducting film can be given with this technique very well, and also a quantitative evaluation should be possible. ${ }^{20}$

At the starting point of the scanning located at the bottom right edge of the film, the flux penetration is similar to that of an homogeneous magnetic field. At the edge, a strong enhancement of magnetic field can be observed because of the demagnetization of the superconductor. Penetration, therefore, starts mostly at the film edges. In the presence of a microstructural defect, e.g., a small scratch, flux can easily penetrate along the defect deeply into the superconducting film and from the end of the defect flux spreads out in a plume-like pattern into the superconducting areas. Along extended linear defects, flux penetration starts in a parabolic shape into the sample similar to the penetration behavior occurring at a linear sample edge. ${ }^{28}$ Different defect structures can clearly be observed at the sample edge [Fig. 5(a)]. Furthermore, also in the center of the YBCO film, some isolated defects are visible. At these defects, some flux has already been pinned during the low field cooling process and has been magnified by increasing the external field. One of the centered defects appears at a magnetic field of $45 \mathrm{mT}$ (arrow 2) and gets more evident at $105 \mathrm{mT}$ [Fig. 5(b), arrow $2]$. The field cooling process could be an advantage for a qualitative defect control due to a pinning of flux at defects during the cool down. In this case, especially defects without connection to the sample edge can probably be easier de- tected at low magnetic fields than in zero field cooled superconductors. A flux penetration into isolated defects in samples after zero field cooling was reported recently. ${ }^{16,19,20}$

As a result of the various defects present in the superconducting film, a complicated network of $d^{+}$lines ${ }^{28,29}$ can be determined. These $d^{+}$lines are caused by a strong bending of the current paths. As flux cannot cross these $d^{+}$lines, they stay dark in the magneto-optical images as can be seen in Fig. 5(b) (e.g., arrows 1). In the presence of defects the course of the $d^{+}$lines is caused by the influence of the defect on the current flow as well as the adjacent defect structure and sample geometry. As a result of the $d^{+}$-line network, the sample can be distinguished in areas with good homogeneity of the electrical properties and with enhanced defect occurrence, which induces a high density of $d^{+}$lines in Fig. 5(b).

Microscopy was used to correlate the magneto-optically observed $j_{c}$ degradations with structural defects in the YBCO thin film. The position with the enhanced linear defect on the 2 in. sample [Fig. 6(a) arrow] was investigated with optical microscopy (OM) and SEM. Fig. 6(a) shows an optical micrograph of the defect in differential interference contrast. The observed defect is about $1 \mathrm{~cm}$ long but only about $5 \mu \mathrm{m}$ wide. This indicates, that a defect detection below the optical resolution of the MOST setup is indeed possible. The defect observed with MOST could not be detected by SEM [inset Fig. 6(a)], because of the fact that there is no trench or heap of material on top of the layer. This indicates that the irregularity is probably due to a scratch in the substrate. The deposition of YBCO covers the scratch and produces a nearly smooth surface, but the structural perfection is destroyed in this area. A detection of the defect by $\mathrm{OM}$ in contrast to SEM was possible, because the YBCO film was rather transparent in the optical range. On the other hand, some scratches detected on the surface of the YBCO film by 
OM could not be associated with structures in the flux pattern of the magneto-optical images. In this case, it seems that the defects have no influence on the superconducting properties because they have no large extension into the layer.

It is obvious that most of the defects detected on the $2 \mathrm{in}$. wafer are near the wafer edge. The nature of these defects is due to a jagged wafer edge caused by a cutting of the substrates.

The 2 in. sample was also investigated by a scanning inductive probe technique ${ }^{30}$ recording the $j_{c}$ values of the sample in a $0.5 \times 0.5 \mathrm{~cm}^{2}$ screen. The scanned $j_{c}$ map is imaged in Fig. 6(b). The critical current density $j_{c}$ varies from $\leqslant 0.5 \mathrm{MA} / \mathrm{cm}^{2}$ at the edge of the sample and two defects to $2.5 \mathrm{MA} / \mathrm{cm}^{2}$ in maximum. A good agreement with the magneto-optical measurements could be observed in those cases of defects having a strong influence on the critical current density. On smaller defects the magneto-optical technique is more sensitive and more complete than the inductive scans. Moreover a defect detection near the sample edges with the inductive $j_{c}$ measurements is not possible.

These results clearly show the advantage of the magneto-optical technique in detecting just all relevant defects having an influence on the critical current density, which is the crucial parameter for high- $T_{c}$ applications.

\section{ACKNOWLEDGMENTS}

The authors would like to thank M. Wallenhorst and $\mathrm{H}$. Dötsch (Universität Osnabrück), as well as I. M. Syvorotka and S. B. Ubizskii (SRC Carat, joint TRANSFORM-project "iron garnet films") for providing the iron garnet films for the magneto-optical investigations. The work was funded within the Siemens Project "HTS-power engineering" by the German ministry BMBF and the Bayerische Forschungsstiftung (FORSUPRA).

${ }^{1}$ P. B. Alers, Phys. Rev. 105, 104 (1957).

${ }^{2}$ W. DeSorbo, Phys. Rev. Lett. 4, 406 (1960).

${ }^{3}$ H. Kirchner, Phys. Lett. 30, 437 (1969).

${ }^{4}$ R. P. Huebener, V. A. Rowe, and R. T. Kampwirth, J. Appl. Phys. 41, 2963 (1970).

${ }^{5}$ M. R. Koblischka and R. J. Wijngaarden, Supercond. Sci. Technol. 8, 199 (1995).
${ }^{6}$ N. Moser, M. R. Koblischka, H. Kronmüller, B. Gegenheimer, and H. Theuss, Physica C 159, 117 (1989).

${ }^{7}$ M. R. Koblischka, N. Moser, B. Gegenheimer, and H. Kronmüller, Physica C 166, 36 (1990).

${ }^{8}$ P. Brüll, D. Kirchgässner, and P. Leiderer, Physica C 182, 339 (1991).

${ }^{9}$ Th. Schuster, M. R. Koblischka, B. Ludescher, N. Moser, and H. Kronmüller, Cryogenics 31, 811 (1991).

${ }^{10}$ V. Bujok, P. Brüll, J. Boneberg, S. Herminghaus, and P. Leiderer, Appl. Phys. Lett. 63, 412 (1993).

${ }^{11}$ P. Leiderer, J. Boneberg, P. Brüll, V. Bujok, and S. Herminghaus, Phys. Rev. Lett. 71, 2646 (1993).

${ }^{12}$ M. V. Indenbom, N. N. Kolesnikov, M. O. Kulakov, I. G. Naumenko, V. I. Nikitenko, A. A. Polyanskii, N. F. Vershinin, and V.K. Vlasko-Vlasov, Physica C 166, 486 (1990).

${ }^{13}$ S. Gotoh and N. Koshizuka, Physica C 176, 300 (1991).

${ }^{14}$ L. A. Dorosinskii, M. V. Indenbom, V. I. Nikitenko, Yu. A. Ossip'yan, A. A. Polyanskii, and V. K. Vlasko-Vlasov, Physica C 203, 149 (1992).

${ }^{15}$ M. V. Indenbom, A. Forkl, B. Ludescher, H. Kronmüller, H.-U. Habermeier, B. Leibold, G. D'Anna, T. W. Li, P. H. Kes, and A. A. Menovsky, Physica C 226, 325 (1994).

${ }^{16}$ J. Eisenmenger, S. Kambach, S. Saleh, A. Tihi, P. Leiderer, M. Wallenhorst, and H. Dötsch J. Low Temp. Phys. 105, 1123 (1996).

${ }^{17}$ M. Kuhn, B. Schey, R. Klarmann, W. Biegel, B. Stritzker, J. Eisenmenger, and P. Leiderer, Physica C 294, 1 (1998).

${ }^{18}$ Th. Schuster, M. R. Koblischka, H. Kuhn, H. Kronmüller, G. Friedl, B. Roas, and L. Schultz. Appl. Phys. Lett. 62, 768 (1993).

${ }^{19}$ M. R. Koblischka, Physica C 259, 135 (1996).

${ }^{20}$ S. A. Govorkov, A. F. Khapikov, B. Heinrich, J. C. Irwins, R. A. Cragg, and A. A. Fife, Supercond. Sci. Technol. 9, 952 (1996).

${ }^{21}$ M. Wallenhorst, M. Niemöller, H. Dötsch, P. Hertel, R. Gerhardt, and B. Gather, J. Appl. Phys. 77, 2902 (1995).

${ }^{22}$ S. B. Ubizskii, I. M. Syvorotka, and P. M. Vetoshko, 7th International Conference on Ferrites, Bordeaux, France, 3-6 Sept. 1996.

${ }^{23}$ S. B. Ubizskii, A. O. Matkovskii, and M. Kuzma, J. Magn. Magn. Mater. 157/158, 279 (1996).

${ }^{24}$ Th. Schuster, H. Kuhn, E. H. Brandt, M. V. Indenbom, M. Kläster, G. Müller-Vogt, H.-U. Habermeier, H. Kronmüller, and A. Forkl, Phys. Rev. B 52, 10375 (1995).

${ }^{25}$ A. Forkl and J. Kronmüller, Phys. Rev. B 52, 16130 (1995).

${ }^{26}$ B. Schey, T. Bollmeier, M. Kuhn, W. Biegel, G. Östreicher, and B. Stritzker, Rev. Sci. Instrum. 69, 474 (1998).

${ }^{27}$ B. Schey, R. Klarmann, W. Biegel, M. Kuhn, and B. Stritzker, Appl. Surf. Sci. 127-29, 540 (1998).

${ }^{28}$ Th. Schuster, M. V. Indenbom, M. R. Koblischka, H. Kuhn, and H. Kronmüller, Phys. Rev. B 49, 3443 (1994).

${ }^{29}$ A. M. Campbell, J. E. Evetts, Critical Currents in Superconductors (Taylor and Frances, London, 1972).

${ }^{30}$ J. H. Claasen, M. E. Reeves, and R. J. Soulen, Rev. Sci. Instrum. 62, 996 (1991). 